\title{
INFLUENCE OF FILLER, MONOMER MATRIX AND SILANE COATING ON COMPOSITE RESIN ADHESION
}

\author{
MIHAELA PĂSTRAVa, MARIOARA MOLDOVAN ${ }^{b}$, \\ ANDREA CHISNOIU ${ }^{a, *}$, CODRUȚA SAROȘI ${ }^{b}$, FILIP MIUȚA ${ }^{b}$, \\ OVIDIU PĂSTRAV ${ }^{a}$, ADA DELEAN $^{\mathrm{a}}$, RADU CHISNOIU ${ }^{\mathrm{a}}$
}

\begin{abstract}
This study compared marginal microleakage in case of two dental composite resins: a consecrated commercial Bis- GMA resin- IPS Empress Direct (Ivoclar Vivadent, Lichtenstein)- group $A$, and a recently introduced Bis- MEPP resin - Gaenial A'Chord (GC R\&D Japan)- group B and to observe, by means of scanning electron microscopy (SEM), the differences between these two restorative materials when using the same adhesive system and restorative layering technique. Microleakage testing scores were higher in IPS Empress group, but the results were not statistically validated. SEM images on group A presented a highly developed hybrid layer, while for group B resin extension intersecting the hybrid layer were observed, having a similar electronic density with the adhesive layer, which demonstrates a continuity of the nanoparticles in depth. On dentincomposite interface increased number of gaps and fissures were observed, comparing to enamel-composite interface. The result from the research showed that the two compared composite resin present similar properties in terms of adhesion and microleakage.
\end{abstract}

Keywords: composite resin, Bis-GMA, Bis-MEPP, silane coating.

\section{INTRODUCTION}

For the past several decades, resin composites have been proposed as an alternative to amalgam, gold and ceramic restorations due to their unique optical, electrical, physical, chemical, and magnetic properties [1].

Fundamentally, the composition of dental composite is represented by an inorganic filler, organic matrix and coupling agent. The fillers represent

a Iuliu Hatieganu University, Faculty of Dental Medicine, 8 Babes str., RO-400012, Cluj-Napoca, Romania.

b Babes-Bolyai University,Raluca Ripan Instutite for Research in Chemistry, 31 Fantanele str., RO-400294, Cluj-Napoca, Romania.

* Corresponding author: maria.chisnoiu@umfcluj.ro. 
the main component which ameliorates the poor mechanical and physical properties of the unfilled resin [2]. Therefore, the ratio of resin/filler content directly affects the material's properties and increasing the filler content will increase wear resistance, strength and will reduce shrinkage properties. The components and ratio of the resin matrix, the shape, size and content of filler and the silane treatment will affect the viscosity improving the handling characteristics of the material, ensuring interlocking between filler particles and interfacial interaction between filler particles and the resin matrix [3].

The organic matrix consists of several monomers, such as BisGMA (2,2-bis[p-(2'-hidroxy-3'-methacryloxypropoxy)phenyl]- propane), UDMA (urethane dimethacrylate), TEGDMA (trietylenglycol dimethacrylate), DMAEMA (dimethylaminoethyl methacrylate- mostly used as an additive as light curing accelerator), BisMEPP (bisphenole A ethoxylate dimethacrylate) and various additives (photoinitiators-camphoroquinone, inhibitors, stabilizers). After the polymerization reaction of the monomer mixture of resin composites, an organic matrix as a three-dimensional cross-linked network is formed [4].

Physical and chemical properties of nanostructured composite materials can be adjusted by controlling the composition and the relative sizes of various components.

The composite nanostructures with High-performance Pulverized CERASMART (HPC) filler and Full- Coverage Silane Coating (FSC) technologies combine properties of the original components but also possess novel and collective performances which are not seen in the original constituents.

The silane coating of the FSC, creates a strong bond between particles in the resin matrix, resulting in a high wear resistance, color retention and excellent radiopacity for long-lasting and natural-looking restorations. HPC filler technology enables improved handling with minimal gaps, providing substantial marginal integrity for virtually eliminating the occurrence of secondary caries. In addition, its ceramic content contributes to the material's durability and ease of handling [5].

The present study was designed to compare marginal microleakage in case of two dental composite resins (a recently introduced Bis- MEPP resin - Gaenial A'Chord (GC R\&D Japan) and a consecrated commercial BisGMA resin- Ips Empress Direct (Ivoclar Vivadent, Lichtenstein), and to observe, by means of scanning electron microscopy (SEM), the differences between these two restorative materials when using the same adhesive system and restorative layering technique.

\section{RESULTS AND DISCUSSION}

Microleakage testing showed higher number of samples with score 0 in group B, comparing to group A. Differences were also observed in case of samples with scores $>0$, but the results were not statistically validated (Figure 1, Table 1). 


\section{Marginal microleakage}

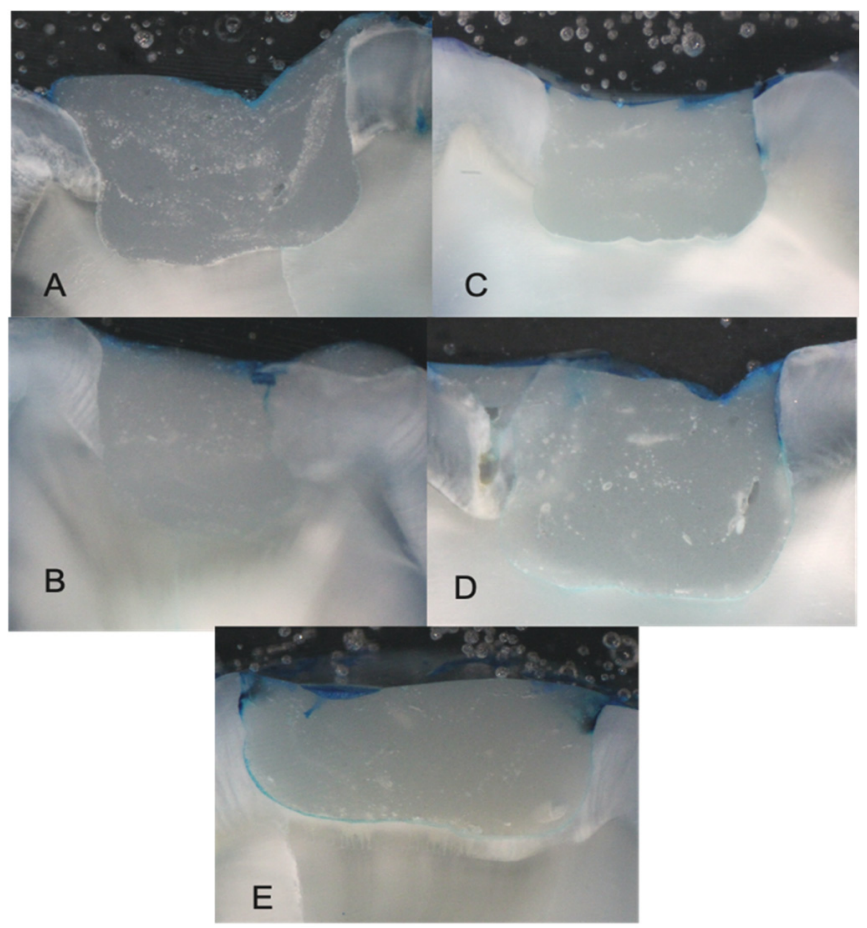

Figure 1. Representative images of degrees of microleakage for Gaenial A'Chord. (Ano evidence of dye penetration, B- $1^{\text {st }}$ degree, C- $2^{\text {nd }}$ degree, $D-3^{\text {rd }}$ degree, E- $4^{\text {th }}$ degree).

Table 1. Microleakage scores of the two composite groups

\begin{tabular}{|c|c|c|c|c|c|c|}
\hline Group & $\begin{array}{c}\text { score 0 } \\
\text { N (\%) }\end{array}$ & $\begin{array}{c}\text { score 1 } \\
\text { N (\%) }\end{array}$ & $\begin{array}{c}\text { score 2 } \\
\mathbf{N}(\%)\end{array}$ & $\begin{array}{c}\text { score 3 } \\
\mathbf{N}(\%)\end{array}$ & $\begin{array}{c}\text { score 4 } \\
\mathbf{N}(\%)\end{array}$ & p \\
\hline A (IPS Empress) & 19 & 6 & 1 & 3 & 1 & \\
& $(63.4)$ & $(20)$ & $(3.33)$ & $(10)$ & $(3.33)$ & \multirow{2}{*}{0.206} \\
\cline { 1 - 5 } B (G-aenial & 22 & 4 & 2 & 1 & 1 & \\
A'Chord) & $(73.34)$ & $(13.34)$ & $(6.67)$ & $(3.33)$ & $(3.33)$ & \\
\hline
\end{tabular}




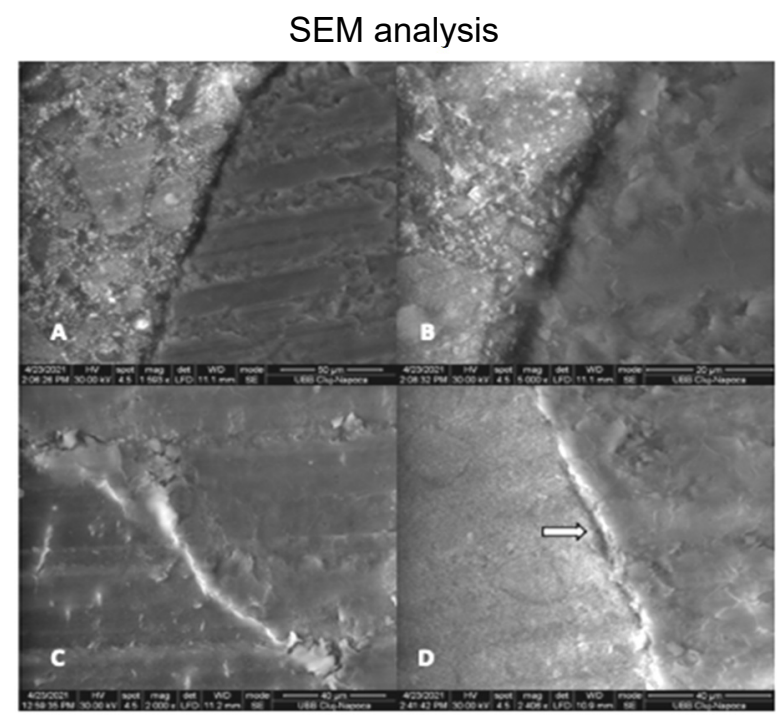

Figure 2. Representative images of the interface composite-dental tissues of the two composite groups. A- Enamel - IPS Empress direct interface; B- Dentin- IPS Empress direct interface; C- Enamel- Gaenial A'Chord interface; D- DentinGaenial A'Chord interface. Arrow- cracks and gaps at material- dentin interface.

SEM analysis was performed to observe the adhesion between the coronary restorative materials and dental tissues for the situations when no microinfiltration was observed (score 0 ), as well as for the situations when the dye penetrated the composite/dental tissue interface (scores $>0$ ) (Figure 2).

In case of samples from group A with score 0 completely adapted adhesive interface was observed, indicating highly developed hybrid layers. SEM images show a uniform and efficient polymerization on the entire restored cavity. The hybrid layer presents a thin, homogenous and electron dense structure with relatively uniform width $(\sim 2 \mu \mathrm{m})$, closely following the dental surface and forming a hybrid layer $(5-10 \mu \mathrm{m})$ which penetrates the dentin (Figure 2-B).

In case of samples from group $B$ and score 0 , resin extension intersecting the hybrid layer have a similar electronic density with the adhesive layer, which demonstrates a continuity of the nanoparticles in depth. The periphery of the dentinal canals was also hybridized and the hybrid layer was extended on the entire depth of the demineralized area (Figure 2-C).

In samples with score higher than 0 , interfacial fissures between the dentin and the hybrid layer were observed on samples from group B. On dentin- composite interface increased number of gaps and fissures were observed, comparing to enamel-composite interface, indicating that dentin adhesion is probably influenced by higher number of factors comparing to enamel adhesion (Figure 2-D). 
In case of Bis GMA resin a porous texture was observed on the SEM photographs, which led to the conclusion that the remaining monomer enabled water molecules to gain better access to the polymer matrix through this porous structure (Figure 2-B).

Characteristics of dental resin composites depend upon several factors including monomer composition, coating chemistry, filler content and irradiation protocol $[6,7,8]$.

The resin matrix influences the properties of dental composites. In the current study the samples contained a Bis-GMA resin and a Bis MEPP resin. Bis-GMA resin in group A present two hydroxy groups which are capable of forming intermolecular bonding. These characteristics were observed on SEM images showing a deep penetration into the dentinal tubules. Previous studies present that the composites based on dimethacrylates with hydroxy groups (Bis-GMA) present improved mechanical properties under dry conditions, comparing to Bis-MEPP resin. Under wet conditions, these composites showed a relatively lower flexural strength than the composites based on dimethacrylates without hydroxy groups (Bis MEPP) [9]. However, Bis-MEPP-based resin presented a lower water sorption and high colour stability comparing with BisGMA resin in a study realized by Mizukami et al [10].

Resin-based dental composites contain filler materials such as barium glass, silica, apatite and a silane coupling agent. The latter enhances the bonding between the filler particles and the resin matrix. The filler particles added to the resin matrix also improve the physical and mechanical properties of the resin composite. Moreover, the addition of fillers reduces volume shrinkage after polymerization, and improves the aesthetic appearance and radiopacity [11].

Generally, surface of inorganic filler is hydrophilic, while resin monomer is hydrophobic. It is important for filler to be treated by silane coupling agent because the interface between filler and resin matrix impact on physical, chemical and mechanical properties of composite. Previous study by Higuchi et al, reported that FSC treated glass filler showed significantly less hydroxygroups than glass filler of control resin and was uniformly covered with silane coupling agents. Results could be from higher chemical reaction rate between $\mathrm{SiOH}$ on the surface of filler and silane coupling agents. FSC has potential to produce higher physical, chemical and mechanical properties on composite product line [12].

From a clinical point of view, composite restoration requires good mechanical properties, such as a high viscosity of the composites and reduced polymerization shrinkage to avoid marginal micro infiltration, but also adequate aesthetics and wear resistance. Therefore, the ratio of resin/filler content directly affects the material's properties. Increasing the filler content results in enhanced wear resistance, strength and reduced shrinkage properties [2]. 
The fillers are the inorganic component of resin composites which are incorporated to enhance the mechanical properties and reduce polymerization shrinkage of resin composites. Pre-polymerized fillers were introduced to improve the filler volume fraction. They are processed using ground cured composite containing a variety of submicron particles [13].

In the present study, SEM images analysis showed that composite resin in group B (containing HPC fillers) which ensured the formation of the hybrid layer presented a deep penetration into the dentinal tubules. The addition of PPFs also aids in reducing the polymerization shrinkage and provides improved polishing when compared to conventionally filled resin composites [14-16].

\section{CONCLUSIONS}

Within the limitations of the current study, the material filler content as well as the resin matrix composition and silane coating influences microleakage of dental composites. Both investigated materials presented similar properties in terms of adhesion and microleakage.

\section{EXPERIMENTAL SECTION}

\section{Preparation of the samples}

Sixty maxillary and mandibular molars were included in the study. The teeth were extracted on orthodontic indication maximum 4 weeks prior to the study. All teeth were caries and filling free, without coronal destruction of other etiology and maintained in distilled water to ensure adequate hydration conditions.

\section{Cavity preparation}

On the occlusal surface of each tooth a Black class I cavity was realized with maximum depth of $3 \mathrm{~mm}$. The preparation was made using 0,12 round turbine burs (MDT, Mc Drill Technology, Parma, Italy) on the enamel layer and 0,14 round tungsten carbide contra-angle burs (Dendia, Dendia $\mathrm{GmbH}$, Austria). The bur was changed after every 5 cavity preparations.

The depth of the cavity was measured from the center of central fissure using UNC 15 probe (Hu-Freidy Mfg. Co. Inc., IL, USA). The width of the cavity was standardized using a divider and scale. 


\section{Sample distribution}

The prepared 60 teeth were randomly divided into 2 groups of thirty teeth each based on the restorative material as Group A - Ips Empress Direct $(n=30)$ and Group B -Gaenial A'Chord $(n=30)$.

Table 2. Chemical composition of the two materials

\begin{tabular}{|l|l|l|}
\hline $\begin{array}{l}\text { Ips Empress Direct } \\
\text { (Ivoclar Vivadent, } \\
\text { Lichtenstein) }\end{array}$ & Nanohybrid & UDMA \\
& & Bis-GMA \\
& & TEGDMA \\
& Barium glass filler $0.4 \mu \mathrm{m}$ \\
& & Prepolymer $1-10 \mu \mathrm{m}$ \\
& Ytterbium trifluoride $100 \mathrm{~nm}$ \\
\hline GC Gaenial A'Chord \\
(Dental Products, Alsip, \\
IL, USA) & Microhybrid & UDMA, \\
& & Bis-MEPP \\
& & TEGDMA \\
& & Silicon dioxide $(16 \mathrm{~nm})$, \\
& Strontium glass $(200 \mathrm{~nm})$, \\
& & Pigment \\
\hline
\end{tabular}

Figure 3 shows the chemical structure of Bis-MEPP and Bis-GMA monomers.<smiles>C=C(C)C(=O)OCCC(=O)OCCOc1ccc(C(C)(C)c2ccc(OCCC(=O)OC(=O)OCc3ccccc3)cc2)cc1</smiles><smiles></smiles>

Figure 3. Chemical structure of Bis-MEPP and Bis-GMA monomers.

\section{Restoration technique}

As adhesive system, we used a 3 steps system, considering that this is the golden standard in dental adhesion. For both groups (A, B), the enamel was etched for 30 seconds and dentin for 15 seconds using $37 \%$ Meta Etchant Gel (Meta Biomed Co. Ltd), then washed and gently dried for approx. 
$5 \mathrm{sec}$. The new G2 - BOND Universal (GC Europe) was applied, according to the manufacturer instructions - first the Primer, for $10 \mathrm{sec}$., with a microbrush and dried for $5 \mathrm{sec}$., then the Bond, spread evenly using a gentle stream of air and light cured for 10 seconds, using a LED light-curing lamp (Translux-Wave, Kulzer). Teeth were then filled (using oblique layering technique and with $1 \mathrm{~mm}$ thick increments and light-curing for 20 seconds) with a nanohybrid composite IPS Empress Direct (Ivoclar, Vivadent, Lichtenstein G) - (group A) and G-aenial A'Chord (GC R\&D, Japan)- (group B).

The shade of the composite was different from the tooth in order to facilitate the assessment. In the end, the restorations were finished and polished with red and yellow ring burs and rubber cups.

All specimens were thermocycled for 1000 cycles $\left(5 / 55^{\circ} \mathrm{C}, 30\right.$ seconds) in Eppendorf Master cycler gradient (Eppendorf AG, Hamburg, Germany). After thermocycling, apices of the teeth were sealed with a layer of sticky wax, and the roots surfaces were covered with two coats of nail polish. All samples were immersed in $2 \%$ methylene blue dye for 24 hours. Following immersion teeth were washed with distilled water then dried. Then they were embedded in acrylic resin (Duracryl Plus, SpofaDental) and, later, sectioned mesiodistally using the microtome (IsoMet TM1000, Buehler, IL, USA). There were selected those teeth slices that have not damages after cutting. Each tooth slice was $1.5 \mathrm{~mm}$ thick.

For marginal microleakage assessment, the cut sections were observed under 20X magnification and the area of maximum dye penetration was considered. For the magnification, a stereomicroscope was used (Zeiss CL 1500 ECO) and each sample was photographed using a digital photo camera (Canon EOS 1300D).

Two examiners scored extent of dye penetration using an ordinal scale (0-4) by consensus. Examiners were blind to material and/or technique used.

The scoring criteria:

$0-$ no evidence of dye penetration

1 - dye penetration along the axial cavity walls up to $1 / 3$

2 - dye penetration along the axial cavity walls up to $2 / 3$

3 - dye penetration along the whole axial cavity wall

4 - dye penetration on the pulpal wall

Samples for scanning electron microscopy (SEM) evaluation were prepared similar as for optical microscopy, in order to evaluate the enamelcomposite and dentin-composite interface, as well as enamel and dentin structure. All measurements were performed using (SEM) QUANTA 133 microscope system (FEI Company USA). Several units of image magnification were used and all registrations were performed on enamel- composite interface and dentincomposite interface. 
The statistical analysis was conducted using MedCalc Statistical Software version 18.11 (MedCalc Software bvba, Ostend, Belgium; http://www.medcalc.org; 2018). Qualitative data were analyzed with Chi-square and Fisher exact test and a $5 \%$ level of significance.

\section{REFERENCES}

1. C. P. Turssi; J. L. Ferracane; K. Vogel. Biomat., 2005, 26, 4932-4937.

2. L. D. Randolph; W. M. Palin; G. Leloup; J. G. Leprince. Dental Mat., 2016 32, 1586-1599.

3. J. H. Lee; C. M. Um; I. Lee. Dental Materials, 2006, 22, 515-526.

4. A. C. Obici; M. A. C. Sinhoreti; E. Frollini; L. C. Sobrinho; S. Consani. Mat. Res. 2004, 7, 605-610.

5. GC Group. Br Dent J 2020, 229, 695.

6. L. Feng; B. I. Suh. Macromol Chem and Phys, 2007, 208, 295-306.

7. N. Emami; K. J. Söderholm. J Mat Sci, 2005, 16, 47-52.

8. A. Peutzfeldt; E. Asmussen. J Dent Res. 2005, 84, 659-62.

9. M. Kawaguchi; T. Fukushima; T. Horibe. Dent Mat J, 2009, 8, 40-45.

10. H. Mizukami; H. Kobayashi; M. Niizuma; Y. Sugai; R. Iketani; Y. Yamada; M. Manabe. IADR/AADR/CADR, 2019, ID: 3652.

11. C.Y.K. Lung; J.P. Matinlinna. Dent Mat, 2012, 28, 467-477.

12. K. Higuchi; D. Usuki; T. Ueno; T. Kumagai. IADR/AADR/CADR, 2019, 3678-3679.

13. J. L. Ferracane; W.V. Giannobile. J Dent Res. 2014, 93, 1185-1186.

14. P. Senawongse; P. Pongprueksa. J Esthet Restor Dent. 2007, 19, 265-273.

15. J. A. Arsecularatne; N. R. Chung; M. Hoffman. Biosurf. Biotribol. 2016, 2, 102-113.

16. M. Moldovan; R. Balazsi; A. Soanca; A. Roman; C. Sarosi; D. Prodan, M. Vlassa; I. Cojocaru; V. Saceleanu; I. Cristescu. Materials, 2019, 12, 2109-2123. 
